\title{
Bose-Einstein Condensation on a Manifold with Nonnegative Ricci Curvature
}

\author{
Levent Akant $^{\dagger}$, Emine Ertuğrul ${ }^{\ddagger}$, \\ Ferzan Tapramaz ${ }^{\S}$, O. Teoman Turgut* \\ Department of Physics, Boğaziçi University \\ 34342 Bebek, Istanbul, Turkey \\ †levent.akant@boun.edu.tr, ${ }^{\ddagger}$ emine.ertugrul@boun.edu.tr,

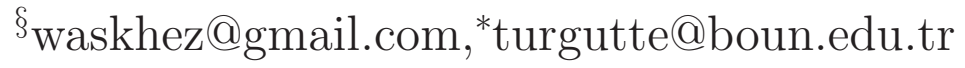

April 2, 2018

\begin{abstract}
The Bose-Einstein condensation for an ideal Bose gas and for a dilute weakly interacting Bose gas in a manifold with nonnegative Ricci curvature is investigated using the heat kernel and eigenvalue estimates of the Laplace operator. The main focus is on the nonrelativistic gas. However, special relativistic ideal gas is also discussed. The thermodynamic limit of the heat kernel and eigenvalue estimates is taken and the results are used to derive bounds for the depletion coefficient. In the case of a weakly interacting gas Bogoliubov approximation is employed. The ground state is analyzed using heat kernel methods and finite size effects on the ground state energy are proposed. The justification of the c-number substitution on a manifold is given.
\end{abstract}




\section{Introduction}

The purpose of this paper is to analyze the depletion coefficient (number density of bosons out of the condensate) and the ground state energy of the Bose-Einstein condensate on a Riemannian manifold with a nonnegative Ricci curvature using global heat kernel and eigenvalue estimates and also the heat kernel asymptotics for the Laplacian on the manifold. The basic observation we make is that both the depletion coefficient and the ground state energy can be expressed in terms of the heat kernel of the Laplacian. Then we exploit this fact and analyze the depletion coefficient and the ground state energy using the above mentioned bounds and asymptotics. Applying our results to the flat space case we derive finite size corrections to the ground state energy of the weakly interacting Bose gas.

We will consider both the ideal gas and the gas with a weak hard-core repulsion [1], [2], [3], [4]. The latter case will be analyzed using the curved space version of the Bogoliubov theory. The Bogoliubov approximation scheme starts with the replacement of the ground state creation and annihilation operators by c-numbers. One then truncates the Hamiltonian, diagonalizes the resulting quadratic Hamiltonian by a Bogoliubov transformation and thus derives the Bogoliubov spectrum. That the first step of this procedure, the c-number substitution, is an exact procedure and not merely an approximation was proven by several authors [7], [8], [9], [10]. In particular, in [8] Lieb et al. have shown that the c-number substitution is a general property of second quantization. The generalization of this property to the Riemannian manifolds will be discussed in the Appendix B. The use of the Neumann boundary conditions for the Bose gas implies that the one particle ground state wave function is constant. Since the ideal gas is noninteracting, the thermodynamic properties are sensitive to the boundary conditions imposed, in a sense all the interaction comes from the boundary. The use of Neumann boundary condition is equally acceptable as the Dirichlet one, the former would mean that the particle flux from the boundary is zero. In our approach the Neumann boundary condition is more natural; in fact, in the case of the ideal gas all the results that we derive using the Neumann boundary conditions can also be obtained for Dirichlet boundary conditions. The homogeneity of the ground state allows us to carry the flat space proof to the curved case. For an extensive review of the Bogoliubov theory in flat space see [11] and references therein (for a curved space version see [12]). For a mathematically rigorous account of Bose-Einstein condensation in flat space see [13], for a more traditional approach see the monographs [14], [15], and for finite temperature gases on curved spaces see [16-20].

The effect of trapping potentials will be discussed elsewhere. We will 
mainly consider the non-relativistic gas. However, in the case of an ideal gas we will discuss the special relativistic generalization, [21-32] as well.

For an ideal gas in a flat manifold the behavior of the chemical potential $\mu$ is crucial for the understanding of the Bose-Einstein condensation. The chemical potential is determined by requiring the constancy of the total number density of the Bose gas. Below a critical temperature $T_{c}$ the chemical potential is of the order of $V^{-1}$ where $V$ is the volume of the gas ${ }^{1}$. This is a direct consequence of the fact that for $T \leq T_{c}$

$$
n_{0}=\lim _{V \rightarrow \infty} \frac{1}{V} \frac{1}{e^{-\beta \mu}-1}=\lim _{V \rightarrow \infty} \frac{1}{V \beta|\mu|}>0 .
$$

Here we assumed that the ground state energy is zero. In the flat space, when we consider a box of typical dimensions given by $L=O\left(V^{1 / 3}\right)$, we have

$$
\epsilon_{\sigma}=O\left(L^{-2}\right) \text { for } \sigma \neq 0 .
$$

Here $\sigma$ 's are the quantum numbers of the gas, with $\sigma=0$ denoting the ground state, and $\epsilon_{\sigma}$ 's are the corresponding energies. Therefore, we see that for $T<T_{c}$, for $\sigma \neq 0$

$$
n_{\sigma} \leq \lim _{V \rightarrow \infty} \frac{1}{V} \frac{1}{e^{\beta \epsilon_{\sigma}}-1}=0 .
$$

So in the thermodynamic limit the ground state is occupied macroscopically, while the excited states are not. As we will see, thanks to eigenvalue bounds on manifolds, this argument remains correct for macroscopically admissible volumes, which we define to be a domain inside the manifold, such that the diameter $D_{M}$ is also $O\left(V^{1 / d}\right)$, where $d$ is the dimension of the manifold. More precisely, on a Riemannian manifold $S$ with a non-negative Ricci curvature the eigenvalues of the Laplacian obey precisely (2) [34], [35] if $L$ is interpreted as the geodesic diameter $D_{M}$ of the confining box which is abstracted as a submanifold $M \subset S$ with boundary. We will assume that the gas obeys Neumann boundary conditions on $\partial M$.

Our main observation is that the number density of the excited particles can be expressed in terms of the trace of the heat kernel on $S$. To see that this is so is not difficult in the case of an ideal gas, but is more involved in the case of a weakly interacting gas. Once we establish this result we will use the heat kernel and eigenvalue bounds of the Laplacian to discuss the Bose-Einstein condensation on $S$. In this paper we will not attempt

\footnotetext{
${ }^{1}$ As the chemical potential is an intensive variable its dependence of $N$ and $V$ is of the form $\mu=f(N / V, T)+O\left(V^{-\lambda}\right)$ where $\lambda$ is a positive coefficient. However at $T=T_{c}$ the leading term vanishes and this gives rise to a volume dependence of the chemical potential.
} 
any comparison with the existing bounds on the depletion coefficient in flat space [36-41]. Our bounds, which are very geometric in character, seem to provide a new class of constraints on the depletion of the condensate. Finally we note the following alternative approaches to the Bose-Einstein condensation in curved spaces [42], [43].

\section{Heat Kernel and Eigenvalue Bounds}

We start by introducing our geometric setting and by summarizing the existing heat kernel and eigenvalue estimates that will play important roles in our subsequent discussions. For the sake of completeness and in order to clarify certain relations between the estimates, we will present more detailed discussion of some of these results in Appendix A, following the mathematics literature.

Let $(S, g)$ be a $d$ dimensional Riemannian manifold with metric $g$ and nonnegative Ricci curvature ${ }^{2}$

$$
\operatorname{Ric}_{S} \geq 0 \text {. }
$$

Let $M$ be a connected open submanifold of $S$ with compact closure and smooth convex boundary $\partial M$. Clearly the Ricci curvature of $M$ is also nonnegative. Let $\Delta$ be the Laplacian of the metric $g$ and $\left\{f_{\sigma}\right\}(\sigma=0,1,2, \ldots)$ be a complete orthonormal set of real (standing wave) square-integrable eigenfunctions of $-\Delta$ on $M$, obeying the Neumann boundary conditions

$$
-\Delta f_{\sigma}=\epsilon_{\sigma} f_{\sigma},\left.\quad \hat{n} \cdot \nabla f_{\sigma}\right|_{\partial K}=0 .
$$

Here $\hat{n}$ is the outward looking unit normal to $\partial M$. The eigenvalues can be ordered as

$$
\epsilon_{0}<\epsilon_{1} \leq \ldots \rightarrow \infty
$$

The ground state is

$$
f_{0}=\frac{1}{\sqrt{V}}
$$

with eigenvalue $\nu_{0}=0$. Here $V$ is the volume of $M$. Connectedness of $M$ implies the uniqueness of the ground state and the existence of the fundamental gap $\epsilon_{1}>0$.

\footnotetext{
${ }^{2} \mathrm{~A}$ symmetric covariant tensor field $T$ of rank 2 is bounded below if there exist a real number $c$ such that for any vector field $X$ on $S$

$$
T(X, X) \geq c g(X, X) .
$$

In this case one writes $T \geq c$.
} 
Note that the reality of the eigenfunctions implies

$$
\int d \mu_{g}(x) f_{\sigma}^{*}(x) f_{\rho}(x)=\int d \mu_{g} f_{\sigma}(x) f_{\rho}(x)=\delta_{\sigma \rho},
$$

and for $\sigma \neq 0$ we also have

$$
\int d \mu_{g}(x) f_{\sigma}(x)=\sqrt{V} \int d \mu_{g} f_{0}(x) f_{\sigma}(x)=0 .
$$

Here $d \mu_{g}$ is the Riemannian volume element corresponding to the metric $g$.

For the Neumann heat kernel on a manifold $M$ with a nonnegative Ricci curvature and diameter $D_{M}{ }^{3}$ one has the following estimates of $\mathrm{Li}$ and Yau [34]

$$
\frac{1}{(4 \pi t)^{d / 2}} V \leq \operatorname{Tr} e^{\Delta t} \leq \tilde{C}(d) g(t)
$$

Here $\tilde{C}(d)$ is a positive constant which depends only on the dimension $d$ and

$$
g(t)= \begin{cases}\left(\frac{D_{M}}{\sqrt{t}}\right)^{d} & \text { if } \sqrt{t} \leq D_{M} \\ 1 & \text { if } \sqrt{t} \geq D_{M} .\end{cases}
$$

A direct consequence of the upper bound for the trace of the heat kernel is the eigenvalue bound [34]

$$
\epsilon_{\sigma} \geq \frac{C(d)}{D_{M}^{2}}(\sigma+1)^{2 / d} \geq \frac{C(d)}{D_{M}^{2}} \sigma^{2 / d},
$$

where $C(d)$ is a positive constant which depends only on the dimension.

On the other hand one also has the following upper bound of Colbois and Maerten [35] for the eigenvalues

$$
\epsilon_{\sigma} \leq B(d)\left(\frac{\sigma}{V}\right)^{2 / d}
$$

Here $B(d)$ is a positive constant which depends only on the dimension.

Using the eigenvalue bounds (12) and (13) we get the following bounds for $\operatorname{Tr}^{\prime} e^{\Delta t}$ (here prime means the ground state is omitted in the trace)

$$
\frac{1}{V} \sum_{\sigma=1}^{\infty} e^{-\frac{t B}{V^{2 / d}} \sigma^{2 / d}} \leq \frac{1}{V} \sum_{\sigma=1}^{\infty} e^{-t \epsilon_{\sigma}} \leq \frac{1}{V} \sum_{\sigma=1}^{\infty} e^{-\frac{t C}{D_{M}^{2}} \sigma^{2 / d}} .
$$

\footnotetext{
${ }^{3}$ The diameter $D_{M}$ of a Riemannian manifold $M$ is given by $D_{M}=\sup \{r(p, q): p, q \in$ $M$ \} where $r$ is the geodesic distance on $M$.
} 
Bounding the series by integrals we get

$$
\int_{1}^{\infty} d x e^{-t B x^{2 / d}} \leq \frac{1}{V} \operatorname{Tr}^{\prime} e^{\Delta t} \leq \frac{D_{M}^{d}}{V} \int_{0}^{\infty} d x e^{-t C x^{2 / d}} .
$$

The integrals can be evaluated explicitly

$$
\frac{1}{B^{d / 2}} \frac{d}{2} \Gamma\left(\frac{d}{2}, \frac{t B}{V^{2 / d}}\right)\left(\frac{1}{t}\right)^{d / 2} \leq \frac{1}{V} \operatorname{Tr}^{\prime} e^{\Delta t} \leq \frac{D_{M}^{d}}{V C^{d / 2}} \Gamma\left(\frac{d}{2}+1\right)\left(\frac{1}{t}\right)^{d / 2} .
$$

Here $\Gamma(x, y)$ is the incomplete gamma function.

Another upper bound for the trace of the heat kernel which holds for large $t$ is [54]

$$
\operatorname{Tr}^{\prime} e^{\Delta t} \leq\left(\operatorname{Tr}^{\prime} e^{\Delta t_{0}}\right) e^{-\epsilon_{1}\left(t-t_{0}\right)} \quad t \geq t_{0} .
$$

Here $t_{0}$ is some fixed time.

\section{Ideal Bose Gas in a Riemannian Manifold}

The single particle free Hamiltonian is taken as

$$
h=-\Delta .
$$

The corresponding many-body Hamiltonian is

$$
H_{0}=\int d \mu_{g} \phi^{\dagger}(x) h \phi(x) .
$$

The thermal averages in the grand-canonical ensemble are given by

$$
\langle O\rangle=\frac{\operatorname{Tr} O e^{-\beta H}}{\operatorname{Tr} e^{-\beta H}} .
$$

Here

$$
H=\int d \mu_{g} \phi^{\dagger}(x)(h-\mu) \phi(x)
$$

and $\mu$ is the chemical potential.

Expanding $H$ in terms of creation-annihilation operators and normal ordering the result we get

$$
H=\sum_{\sigma}\left(\epsilon_{\sigma}-\mu\right) a_{\sigma}^{\dagger} a_{\sigma}
$$

Thus the mean occupation numbers are given exactly as in the flat case

$$
N_{\sigma}=\left\langle a_{\sigma}^{\dagger} a_{\sigma}\right\rangle=\frac{1}{e^{\beta\left(\epsilon_{\sigma}-\mu\right)}-1},
$$


where we have taken, as usual, $\mu<0$. Clearly, $N_{0}$ is singular at $\mu=0$. As in the flat case, fixing the total number of particles allows us to determine $\mu$. To maximize the occupation of each level, we should let $\mu \rightarrow 0^{-}$. However, that is a delicate limit, the chemical potential is not strictly zero, the macroscopic occupation of the ground state again leads to $\mu=O\left(V^{-1}\right)$ so that we find a well-defined thermodynamic limit. Note that

$$
N=\sum_{\sigma} \frac{1}{e^{\beta\left(\epsilon_{\sigma}-\mu\right)}-1}
$$

does not scale in a simple way to determine the chemical potential in a simple way even in the continuum limit, since the density of states may be a complicated expression in general.

Finite Volume: First note that

$$
n_{\sigma}=\frac{N_{\sigma}}{V}=\frac{1}{V} \frac{e^{-\beta\left(\epsilon_{\sigma}-\mu\right)}}{1-e^{-\beta\left(\epsilon_{\sigma}-\mu\right)}}=\frac{1}{V} \sum_{k=1}^{\infty} e^{k \beta \mu} e^{-k \beta \epsilon_{\sigma}},
$$

and consequently,

$$
\begin{aligned}
n_{e}(T):=\sum_{\sigma \neq 0} n_{\sigma} & =\frac{1}{V} \sum_{\sigma \neq 0} \sum_{k=1}^{\infty} e^{k \beta \mu} e^{-k \beta \epsilon_{\sigma}} \\
& =\frac{1}{V} \sum_{k=1}^{\infty} e^{k \beta \mu} \operatorname{Tr}^{\prime} e^{-k \beta h} .
\end{aligned}
$$

Here $\operatorname{Tr}^{\prime}$ denotes the trace with the ground state omitted. We employ (17) to study the low temperature behavior of $N_{e}(T)$. Fixing $\beta_{0}$ such that $\beta>\beta_{0}$ we see that

$$
n_{e}(T) \leq \frac{1}{V} e^{\epsilon_{1} \beta_{0}}\left(\operatorname{Tr}^{\prime} e^{-h \beta_{0}}\right) \sum_{k=1}^{\infty} e^{-k \epsilon_{1} \beta}=\frac{1}{V}\left(\operatorname{Tr}^{\prime} e^{-h \beta_{0}}\right) \frac{e^{\epsilon_{1} \beta_{0}}}{e^{\epsilon_{1} \beta}-1} .
$$

So by choosing $T$ low enough we can make $n_{e}$ less than any preassigned value of $n$. Then the particles in excess, whose density is $n-n_{e}$, form condensate in the ground state.

The above analysis is independent of the dimension of the manifold $M$ and the bound on the Ricci curvature. However, the right hand side of (27) is in general divergent as $V \rightarrow \infty$. So the bound is useless in the thermodynamic limit. 
Thermodynamic Limit in Terms of the Heat Kernel: Using the upper bound given in (11) we see that

$$
\begin{aligned}
n_{e}(T) & \leq \frac{\tilde{C}(d)}{V} \sum_{k=1}^{\infty} e^{-k \beta|\mu|} g(k \beta) \\
& \leq \frac{\tilde{C}(d) D_{M}^{d}}{V} \frac{1}{\beta^{d / 2}} \sum_{k=1}^{\left[D_{M}^{2} / \beta\right]} \frac{e^{-k \beta|\mu|}}{k^{d / 2}}+\frac{\tilde{C}(d)}{V} \sum_{k=\left[D_{M}^{2} / \beta\right]+1}^{\infty} e^{-k \beta|\mu|} .
\end{aligned}
$$

Here square brackets mean integer part. Now the second term is just a geometric series whose sum is

$$
\frac{\tilde{C}(d)}{V} \frac{e^{-\left(\left[\frac{D_{M}^{2}}{\beta}\right]+1\right) \beta|\mu|}}{1-e^{-\beta|\mu|}} .
$$

Using $\mu=O\left(V^{-1}\right)$ we see that this goes to zero in the thermodynamic limit $V, D_{M} \rightarrow \infty$.

Our basic assumption regarding the thermodynamic limit will be the following asymptotic relation between the volume and the diameter of our box $M$

$$
D_{M}=O\left(V^{1 / d}\right) \quad \text { as } \quad V \rightarrow \infty .
$$

This is a nontrivial condition and it is not so obvious if we can satisfy this on a Ricci nonnegative manifold. If we have a strictly positive lower bound for the Ricci, by Myers' theorem the manifold necessarily becomes compact. In our case, due to the Bishop-Gromov volume coomparison theorem, the geodesic balls in a Ricci non-negative space cannot have volumes growing faster than the flat case [5]. This is important since, a natural set of boxes to consider would be geodesic balls. It is known that on a complete Riemannian manifold $\mathcal{M}$ of dimension $n$, with nonnegative Ricci , there is a constant $\epsilon(n)$ such that if for some point $p$ we have

$$
\operatorname{Vol} B_{r}(p) \geq(1-\epsilon(n)) \Omega_{n} r^{n}
$$

for all $r$, where $\Omega_{n}$ refers to the volume of the standart unit $n$-ball, then $\mathcal{M}$ is diffeomorphic to the Euclidean space, but not necessarily isometric to it. So clearly there are some interesting examples within our class of manifolds. For further results and references related to this subject we refer to the review article [6].

Under this assumption, in the thermodynamic limit we get

$$
n_{e}(T) \leq \tilde{C}(d) \frac{A}{\beta^{d / 2}} \sum_{k=1}^{\infty} \frac{e^{-k \beta|\mu|}}{k^{d / 2}} \leq \tilde{C}(d) \frac{A}{\beta^{d / 2}} \sum_{k=1}^{\infty} \frac{1}{k^{d / 2}}=\tilde{C}(d) \frac{A}{\beta^{d / 2}} \zeta\left(\frac{d}{2}\right)
$$


Here

$$
A=\lim _{V \rightarrow \infty} \frac{D_{M}^{d}}{V} .
$$

For $d \geq 3 n_{e}(T)$ is clearly finite and vanishes as $T \rightarrow 0$.

For $d=2$ the above bound is of no use in the thermodynamic limit. In order to deduce the behavior of $n_{e}(T)$ at $d=2$ we use the lower bound given in (11).

$$
\begin{aligned}
n(T) & =\frac{1}{V} \sum_{k=1}^{\infty} e^{-k \beta|\mu|}\left(\operatorname{Tr} e^{-k \beta h}\right) \\
& =\frac{1}{V} \sum_{k=1}^{\infty} e^{-k \beta|\mu|} \operatorname{Tr} e^{-k \beta h} \\
& \geq \frac{1}{(4 \pi \beta)^{d / 2}} \sum_{k=1}^{\infty} \frac{e^{-k \beta|\mu|}}{k^{d / 2}} .
\end{aligned}
$$

By a simple integral test the series is seen to be larger than

$$
\int_{1}^{\infty} d x \frac{e^{-x \beta|\mu|}}{x^{d / 2}}=(\beta|\mu|)^{d / 2-1} \int_{\beta|\mu|}^{\infty} d y \frac{e^{-y}}{y^{d / 2}}=(\beta|\mu|)^{d / 2-1} \Gamma(1-d / 2, \beta|\mu|) .
$$

Here $\Gamma(x, y)$ is the incomplete gamma function. If it was the case that $\mu=$ $O\left(V^{-1}\right)$ then for $d=2$ we would have a divergent $n$, which is a contradiction. Thus $\mu \neq O\left(V^{-1}\right)$ and condensation does not take place in two dimensions.

Thermodynamic Limit in Terms of Eigenvalues: Now using the bounds (12) and (13) on the eigenvalues of the Laplacian we get

$$
\sum_{\sigma=1}^{\infty} \frac{1}{V} \frac{1}{e^{\beta\left(\frac{B}{V^{2 / d}} \sigma^{2 / d}-\mu\right)}-1} \leq n_{e} \leq \sum_{\sigma=1}^{\infty} \frac{1}{V} \frac{1}{e^{\beta\left(\frac{C}{D^{2}} \sigma^{2 / d}-\mu\right)}-1} .
$$

But $\mu=O\left(V^{-1}\right)$ and the depletion coefficient can be bound in the $V, D_{M} \rightarrow \infty$ limit as

$$
\int_{0}^{\infty} d x \frac{1}{e^{\beta B x^{2 / d}}-1} \leq n_{e} \leq A \int_{0}^{\infty} d x \frac{1}{e^{\beta C x^{2 / d}}-1}
$$

or after a change of variable

$$
\frac{d}{2(\beta B)^{d / 2}} \int_{0}^{\infty} d \varepsilon \frac{\varepsilon^{\frac{d}{2}-1}}{e^{\varepsilon}-1} \leq n_{e} \leq \frac{A d}{2(\beta C)^{d / 2}} \int_{0}^{\infty} d \varepsilon \frac{\varepsilon^{\frac{d}{2}-1}}{e^{\varepsilon}-1} .
$$


Note that the numerator of the integrand is in accordance with the Weyl asymptotic formula for the eigenvalue density of the Laplacian (see e.g. [55]). Since

$$
\int_{0}^{\infty} d \varepsilon \frac{\varepsilon^{\frac{d}{2}-1}}{e^{\varepsilon}-1}=\Gamma\left(\frac{d}{2}\right) \zeta\left(\frac{d}{2}\right)
$$

we get

$$
\frac{d}{2(\beta B)^{d / 2}} \Gamma\left(\frac{d}{2}\right) \zeta\left(\frac{d}{2}\right) \leq n_{e} \leq \frac{A d}{2(\beta C)^{d / 2}} \Gamma\left(\frac{d}{2}\right) \zeta\left(\frac{d}{2}\right)
$$

On the other hand in flat space

$$
n_{e}^{\text {flat }}=S(d) \frac{1}{\beta^{d / 2}} \Gamma\left(\frac{d}{2}\right) \zeta\left(\frac{d}{2}\right) .
$$

Here $S(d)$ is the usual density of states factor in $\mathbf{R}^{d}$

$$
S(d)=V\left(\mathbf{S}^{d-1}\right) \frac{m^{d / 2}}{2^{d / 2+1} \pi^{d}} .
$$

So

$$
\left(\frac{d}{2 S(d) B^{d / 2}}\right) n_{e}^{\text {flat }} \leq n_{e} \leq\left(\frac{A d}{2 S(d) C^{d / 2}}\right) n_{e}^{\text {flat }} .
$$

Thus we see that $n_{e}$ is divergent for $d \leq 2$ and convergent for $d>2$. Moreover for $d>2, n_{e} \rightarrow 0$ as $T \rightarrow 0$ and we have Bose-Einstein condensation at low temperatures. In fact the critical temperature can be bound as

$$
\left[\frac{A d}{2 n C^{d / 2}} \Gamma\left(\frac{d}{2}\right) \zeta\left(\frac{d}{2}\right)\right]^{-2 / d} \leq k_{B} T_{c} \leq\left[\frac{d}{2 n B^{d / 2}} \Gamma\left(\frac{d}{2}\right) \zeta\left(\frac{d}{2}\right)\right]^{-2 / d} .
$$

\section{Relativistic Ideal Gas}

In this section we will discuss briefly the use of the heat kernel method in the case of a relativistic ideal Bose gas. Consider a $1+3$ dimensional manifold with an ultra-static metric

$$
d s^{2}=-d t^{2}+h_{i j} d x^{i} d x^{j}
$$

Here $\partial_{t} h_{i j}=0$. The Ricci curvature tensor ${ }^{3} R_{i j}$ of the space-like slices is assumed to be nonnegative. The equilibrium number density of the excited particles is given by (see e.g. [33])

$$
n_{e}(T)=\frac{1}{V} \sum_{\sigma \neq 0}\left[\frac{1}{e^{\left(\beta^{2} \lambda_{\sigma}+\beta^{2} m^{2}\right)^{1 / 2}-\beta \mu}-1}-\frac{1}{e^{\left(\beta^{2} \lambda_{\sigma}+\beta^{2} m^{2}\right)^{1 / 2}+\beta \mu}-1}\right] .
$$


Here $\lambda_{\sigma}$ 's are the eigenvalues of the Neumann problem for $-{ }^{(3)} \Delta$ on the space-like slices of our metric. Using the subordination identity

$$
e^{-b \sqrt{x}}=\frac{b}{2 \sqrt{\pi}} \int_{0}^{\infty} \frac{d s}{s^{3 / 2}} e^{-\frac{b^{2}}{4 s}} e^{-s x}
$$

we see that

$$
n_{e}(T)=\sum_{k=-\infty}^{\infty} \int_{0}^{\infty} \frac{d s}{s^{3 / 2}} \frac{k}{2 \sqrt{\pi}} e^{\frac{-k^{2}}{4 s}+k \beta \mu} \frac{1}{V}\left(\operatorname{Tr}^{\prime} e^{s \beta^{2} \Delta}\right) e^{-s \beta^{2} m^{2}},
$$

which could also be written as

$$
n_{e}(T)=\sum_{k=1}^{\infty} \int_{0}^{\infty} \frac{d s}{s^{3 / 2}} \frac{k}{\sqrt{\pi}} e^{-\frac{k^{2}}{4 s}} \sinh (k \beta \mu) \frac{1}{V}\left(\operatorname{Tr}^{\prime} e^{s \beta^{2} \Delta}\right) e^{-s \beta^{2} m^{2}} .
$$

Now using (16) in the thermodynamic limit and noticing

$$
\int_{0}^{\infty} \frac{d s}{s^{3}} e^{-\frac{k^{2}}{4 s}-s \beta^{2} m^{2}}=\frac{8 \beta^{2} m^{2}}{k^{2}} K_{2}(k \beta m),
$$

we get

$$
\frac{3 m^{2}}{B^{3 / 2} \beta} \sum_{k \neq 0} \frac{e^{k \beta \mu}}{k} K_{2}(k \beta m) \leq n_{e}(T) \leq \frac{3 A m^{2}}{C^{3 / 2} \beta} \sum_{k \neq 0} \frac{e^{k \beta \mu}}{k} K_{2}(k \beta m) .
$$

Here $K_{2}$ is the modified Bessel function of the second kind. We recall that $K_{\nu}$ has the following integral representation for $\nu>0$ and $x>0$,

$$
K_{\nu}(x)=\left(\frac{\pi}{2 x}\right)^{1 / 2} \frac{e^{-x}}{\Gamma(\nu+1 / 2)} \int_{0}^{\infty} d s e^{-s} s^{\nu-1 / 2}\left(1+\frac{s}{2 x}\right)^{\nu-1 / 2} .
$$

Using simple estimates we get an upper bound,

$$
K_{2}(x)<\frac{4}{3 \sqrt{2 x}} e^{-x}\left[\Gamma(5 / 2)+\frac{\Gamma(7 / 2)}{x}+\frac{\Gamma(9 / 2)}{4 x^{2}}\right] .
$$

Moreover, by restricting the above sum to only the positive values of $k$, we get an upper bound. Note that the terms of this series are all decreasing, and we also emphasize that $\mu<m$ and the maximum is achieved as $\mu \rightarrow m^{-}$.

$$
\begin{aligned}
n_{e}(T)< & \frac{3 A m^{2}}{C^{3 / 2} \beta} e^{\beta \mu} K_{2}(\beta m) \\
& +\frac{4 A m^{2}}{C^{3 / 2} \beta} \int_{1}^{\infty} d x \frac{e^{-x \beta(m-\mu)}}{(\beta m)^{1 / 2} x^{3 / 2}}\left[\Gamma(5 / 2)+\frac{\Gamma(7 / 2)}{x \beta m}+\frac{\Gamma(9 / 2)}{4(\beta m x)^{2}}\right] .
\end{aligned}
$$


All these terms are finite when we take the limit $\mu \rightarrow m^{-}$, hence beyond a critical density for a given temperature, we will have Bose-Einstein condensation. It is interesting to test $T \rightarrow 0^{+}$limit as well. We see that as we let $\beta \rightarrow \infty$, the upper bound on the Bessel funtion implies that none of the excited levels could be occupied.

Let us also remark on the two dimensional case, following a similar analysis, we have

$\frac{m^{3 / 2}}{B \beta^{1 / 2}} \sum_{k=1} \frac{\sinh (k \beta \mu)}{k^{1 / 2}} K_{3 / 2}(k \beta m) \leq n_{e}(T) \leq \frac{A m^{3 / 2}}{C \beta^{1 / 2}} \sum_{k=1} \frac{\sinh (k \beta \mu)}{k^{1 / 2}} K_{3 / 2}(k \beta m)$.

Note that the Bessel function $K_{3 / 2}$ is given by

$$
K_{3 / 2}(x)=\left(\frac{\pi}{2 x}\right)^{1 / 2} \frac{e^{-x}}{\Gamma(3 / 2)}\left[1+\frac{1}{x}\right]>\left(\frac{\pi}{2 x}\right)^{1 / 2} \frac{e^{-x}}{\Gamma(3 / 2)} .
$$

This is a monotonically decreasing function hence, integral from 1 to $\infty$ provides a lower bound;

$$
\frac{m^{3 / 2}}{B \beta} \int_{1}^{\infty} d x \sinh (x \beta \mu) \frac{e^{-x \beta m}}{x} \leq n_{e}(T) .
$$

For finite $\beta$ this integral is ultraviolet divergent if we let $\mu \rightarrow m^{-}$. Hence there is no need for condensation in two dimensions.

We note the alternative expression for the excited density:

$$
\frac{\partial}{\partial(\beta \mu)} \sum_{k=-\infty}^{\infty} \int_{0}^{\infty} \frac{d s}{s^{3 / 2}} \frac{1}{2 \sqrt{\pi}} e^{\frac{-k^{2}}{4 s}+k \beta \mu}\left(\frac{1}{V} \operatorname{Tr}^{\prime} e^{s \beta^{2} \Delta}\right) e^{-s \beta^{2} m^{2}}
$$

Again, we note that the $k$ sum gives the trace of the heat kernel on $S^{1}$ coupled to the vector potential $a=2 s \beta \mu d \theta$. Thus we get

$$
n_{e}(T)=\frac{\partial}{\partial(\beta \mu)} \int_{0}^{\infty} \frac{d s}{s^{3 / 2}} e^{-s \beta^{2}\left(m^{2}-\mu^{2}\right)}\left(\frac{1}{V} \operatorname{Tr}^{\prime} e^{s \beta^{2} \Delta}\right)\left(\frac{\sqrt{\pi}}{2 \pi} \operatorname{Tr}^{\prime} e^{\frac{1}{4 s} \Delta_{S^{1}}(a)}\right)
$$

Here

$$
\Delta_{S^{1}}(a)=-\left(-i \frac{d}{d \theta}-a\right)^{2}
$$

is the Laplacian on $S^{1}$ coupled to the vector potential $a$ ( $\theta$ is the coordinate on $S^{1}$ ). 
On the other hand the $k$ sum can also be calculated using the Jacobi theta function of the third kind

$$
\sum_{k=-\infty}^{\infty} e^{-\frac{k^{2}}{4 t}+k \beta \mu}=\theta_{3}\left(\frac{\beta \mu}{2 i}, e^{-\frac{1}{4 t}}\right) .
$$

Thus we get the alternative expression

$$
n_{e}(T)=\frac{\partial}{\partial(\beta \mu)} \int_{0}^{\infty} \frac{d s}{s^{3 / 2}} \frac{1}{2 \sqrt{\pi}} e^{-s \beta^{2} m^{2}}\left(\frac{1}{V} \operatorname{Tr}^{\prime} e^{s \beta^{2} \Delta}\right) \theta_{3}\left(\frac{\beta \mu}{2 i}, e^{-\frac{1}{4 s}}\right) .
$$

\section{Bogoliubov Theory on a Compact Rieman- nian Manifold}

The many-body Hamiltonian with a hard-core repulsive potential is given by [1], [2], [3], [4]

$$
H^{\prime}=\int d \mu_{g}\left[\phi^{\dagger}(x) h \phi(x)+\frac{u_{0}}{2} \phi^{\dagger}(x) \phi^{\dagger}(x) \phi(x) \phi(x)\right] \quad\left(u_{0}>0\right) .
$$

It is convenient to include the chemical potential in the Hamiltonian and define

$$
H=H^{\prime}-\mu N .
$$

We will study this Hamiltonian and the Bose-Einstein condensation by applying the curved space version of the standard Bogoluibov theory [1] to it. In flat space the Bogoliubov approximation consists of three steps. First, one replaces the zero energy (ground state) creation and annihilation operators by their coherent state lower symbols and then one expands the Hamiltonian around the c-number background obtained in the first step, ignoring third and higher order terms in the fluctuation. The resulting Hamiltonian is quadratic in the creation and annihilation operators but not diagonal. The final step is the diagonalization of this Hamiltonian by the Bogoluibov transformation. The justification of the c-number replacement was first given in [7] and more recently (and with less assumptions) in [8] (see also [9], [10]). In Appendix $\mathrm{B}$ we will show that on any Riemannian manifold (without any restriction on the Ricci curvature), thanks to the Neumann boundary condition which implies the constancy of the ground state wave-function, the c-number substitution is justified in a way similar to the flat case discussed in [8]. On the other hand, in the semiclassical approximation of the Hamiltonian the Neumann boundary condition and the constancy of the wave-function again play 
important roles. Finally the Bogoluibov transformation which is a purely algebraic manipulation proceeds in the usual way.

Therefore our starting point will be the expansion of the field operator $\phi(x)$ around the background

$$
\phi_{0}(x)=\sqrt{N_{0}} f_{0}=\sqrt{\frac{N_{0}}{V}}=\sqrt{n_{0}}
$$

as

$$
\phi(x)=\phi_{0}(x)+\eta(x)
$$

with

$$
\eta(x)=\sum_{\sigma \neq 0} a_{\sigma} f_{\sigma}
$$

Assuming the quantum fluctuations to be small we can approximate $H^{\prime}$ as

$$
\begin{aligned}
H_{e f f}^{\prime}= & \int d \mu_{g}\left(\phi_{0}(x) h \eta(x)+\eta^{\dagger}(x) h_{0} \eta(x)+\frac{u_{0}}{2}\left[\phi_{0}^{4}+2 \phi_{0}^{3}\left(\eta^{\dagger}(x)+\eta(x)\right)\right.\right. \\
& \left.\left.\phi_{0}^{2}\left(\eta^{2}(x)+\eta^{\dagger 2}(x)+4 \eta^{\dagger}(x) \eta(x)\right)\right]\right) .
\end{aligned}
$$

Expanding in terms of creation and annihilation operators and using (9) and (10) we get

$$
H_{e f f}^{\prime}=\frac{u_{0} n_{0}^{2} V}{2}+\sum_{\sigma \neq 0}\left[\left(\epsilon_{\sigma}+2 u_{0} n_{0}\right) a_{\sigma}^{\dagger} a_{\sigma}+\frac{u_{0} n_{0}}{2}\left(a_{\sigma}^{2}+a_{\sigma}^{\dagger}\right)\right] .
$$

Similarly, the number operator is given by

$$
\begin{aligned}
N & =\int d \mu_{g}(x) \phi^{\dagger}(x) \phi(x) \\
& =\int d \mu_{g}(x)\left[\phi_{0}^{2}+\phi_{0}\left(\eta^{\dagger}(x)+\eta(x)\right)+\eta^{\dagger}(x) \eta(x)\right] \\
& =n_{0} V+\sum_{\sigma \neq 0} a_{\sigma}^{\dagger} a_{\sigma} .
\end{aligned}
$$

So,

$$
H_{e f f}^{\prime}-\mu N=\frac{u_{0} n_{0}^{2} V}{2}-\mu n_{0} V+\sum_{\sigma \neq 0}\left[\left(\epsilon_{\sigma}+2 u_{0} n_{0}-\mu\right) a_{\sigma}^{\dagger} a_{\sigma}+\frac{u_{0} n_{0}}{2}\left(a_{\sigma}^{2}+a_{\sigma}^{\dagger 2}\right)\right] .
$$

For a fixed $n_{0}$ the thermodynamic pressure

$$
\frac{1}{V} \ln \operatorname{Tr}^{\prime} e^{-\beta\left(H_{e f f}^{\prime}-\mu N\right)}
$$


is maximized at the zeroth order in $\eta$ by $\mu=n_{0} u_{0}$. Here $\operatorname{Tr}^{\prime}$ means that the trace is taken over the states with no quanta in the $f_{0}$ mode. With this value of $\mu$ we get

$$
H_{e f f}^{\prime}-\mu N=-\frac{u_{0} n_{0}^{2} V}{2}+\sum_{\sigma \neq 0}\left[\left(\epsilon_{\sigma}+u_{0} n_{0}\right) a_{\sigma}^{\dagger} a_{\sigma}+\frac{u_{0} n_{0}}{2}\left(a_{\sigma}^{2}+a_{\sigma}^{\dagger 2}\right)\right] .
$$

Therefore the thermal averages can be calculated using the effective Hamiltonian

$$
H_{e f f}=\sum_{\sigma \neq 0}\left[\left(\epsilon_{\sigma}+u_{0} n_{0}\right) a_{\sigma}^{\dagger} a_{\sigma}+\frac{u_{0} n_{0}}{2}\left(a_{\sigma}^{2}+a_{\sigma}^{\dagger 2}\right)\right],
$$

which can be diagonalized by the Bogoliubov transformation

$$
a_{\sigma}=\left(\sinh \xi_{\sigma}\right) b_{\sigma}^{\dagger}+\left(\cosh \xi_{\sigma}\right) b_{\sigma},
$$

where

$$
\begin{aligned}
\lambda_{\sigma} & :=\epsilon_{\sigma}+u_{0} n_{0}=\omega_{\sigma} \cosh 2 \xi_{\sigma}, \\
u_{0} n_{0} & =-\omega_{\sigma} \sinh 2 \xi_{\sigma}, \\
\omega_{\sigma} & :=\sqrt{\left(\epsilon_{\sigma}+u_{0} n_{0}\right)^{2}-\left(u_{0} n_{0}\right)^{2}} .
\end{aligned}
$$

The last equation is the curved space analog of the Bogoliubov dispersion relation. The resulting diagonal Hamiltonian is

$$
\begin{aligned}
H_{e f f} & =\sum_{\sigma \neq 0}\left[\lambda_{\sigma} \cosh 2 \xi_{\sigma}+u_{0} n_{0} \sinh 2 \xi_{\sigma}\right] b_{\sigma}^{\dagger} b_{\sigma}+E_{0} \\
& =\sum_{\sigma \neq 0} \omega_{\sigma} b_{\sigma}^{\dagger} b_{\sigma}+E_{0},
\end{aligned}
$$

where $E_{0}$ is the ground state energy

$$
\begin{aligned}
E_{0} & =-\frac{1}{2} \sum_{\sigma=1}^{\infty}\left(\lambda_{\sigma}-\omega_{\sigma}\right) \\
& =-\frac{1}{2} \sum_{\sigma=1}^{\infty} \frac{\left(u_{0} n_{0}\right)^{2}}{2} \frac{1}{\epsilon_{\sigma}}+O\left(\frac{1}{\epsilon_{\sigma}^{2}}\right) .
\end{aligned}
$$

Now using the lower eigenvalue bound (12) we see that for $k \geq 2$ and $d=3,2$

$$
\sum_{\sigma=1}^{\infty} \frac{1}{\epsilon_{\sigma}^{k}}
$$


is convergent. On the other hand, using (13) we see that the $k=1$ sum is divergent for $d=3,2$.

Thus we arrive at the renormalized ground state energy.

$$
E_{0}=-\frac{1}{2} \sum_{\sigma=1}^{\infty}\left[\left(\epsilon_{\sigma}+u_{0} n_{0}\right)-\sqrt{\epsilon_{\sigma}^{2}+2 u_{0} n_{0} \epsilon_{\sigma}}-\frac{\left(u_{0} n_{0}\right)^{2}}{2 \epsilon_{\sigma}}\right] .
$$

Indeed, a closer inspection of the original Hamiltonian given by equation (72) reveals that the bare coupling constant should be replaced by a renormalized coupling constant. The bare coupling can be solved order by order in terms of the renormalized coupling constant $u_{0}^{r}$, to make the effective potential welldefined, and only the first term of (72), within perturbation theory, should be modified by this subtraction. This gives us the desired term. From now on, not to complicate matters we will interpret $u_{0}$ as the renormalized coupling constant.

Finally, the ground state is the coherent state (see e.g. [44])

$$
|\Omega\rangle=\mathcal{N} \prod_{\sigma=1}^{\infty} e^{-\frac{1}{2} \tanh \xi_{\sigma} a_{\sigma}^{\dagger 2}}|0\rangle
$$

with the normalization constant

$$
\mathcal{N}=\prod_{\sigma=1}^{\infty} \frac{1}{\sqrt{\cosh \xi_{\sigma}}}=\left[\prod_{\sigma=1}^{\infty} 1-\frac{\lambda_{\sigma}-\omega_{\sigma}}{\lambda_{\sigma}+\omega_{\sigma}}\right]^{1 / 4} .
$$

Since $0 \leq\left(\lambda_{\sigma}-\omega_{\sigma}\right)\left(\lambda_{\sigma}+\omega_{\sigma}\right)^{-1}<1$ the convergence of the product is equivalent to the convergence of the series

$$
\sum_{\sigma=1}^{\infty} \frac{\lambda_{\sigma}-\omega_{\sigma}}{\lambda_{\sigma}+\omega_{\sigma}}
$$

But,

$$
\sum_{\sigma=1}^{\infty} \frac{\lambda_{\sigma}-\omega_{\sigma}}{\lambda_{\sigma}+\omega_{\sigma}} \leq \sum_{\sigma=1}^{\infty} \frac{\lambda_{\sigma}-\omega_{\sigma}}{\epsilon_{\sigma}}=\sum_{\sigma=1}^{\infty} \frac{\lambda_{\sigma}-\omega_{\sigma}-\frac{\left(u_{0} n_{0}\right)^{2}}{2 \epsilon_{\sigma}}}{\epsilon_{\sigma}}+\sum_{\sigma=1}^{\infty} \frac{\left(u_{0} n_{0}\right)^{2}}{2 \epsilon_{\sigma}^{2}} .
$$

By the eigenvalue estimates given above, the last series is convergent. On the other hand for $\sigma$ large enough

$$
\frac{\lambda_{\sigma}-\omega_{\sigma}-\frac{\left(u_{0} n_{0}\right)^{2}}{2 \epsilon_{\sigma}}}{\epsilon_{\sigma}} \leq \lambda_{\sigma}-\omega_{\sigma}-\frac{\left(u_{0} n_{0}\right)^{2}}{2 \epsilon_{\sigma}} .
$$

Combining this with our discussion of the ground state energy we see that the first series is convergent as well. Thus we conclude $\mathcal{N}<\infty$. 


\section{Ground State Energy and Finite Size Ef- fects}

To understand the ground state energy for three dimensions better, we will now express it in terms of the heat kernel. Consider the first two terms before the renormalization -there is $-1 / 2$ in front of the whole expression. Let's write $s$ for $\epsilon_{\sigma}$ and $a$ for $u_{0} n_{0}$, for simplicity. When we get $e^{-s t}$ the sum over $\sigma$ gives us $\operatorname{Tr} e^{\Delta t}$. Consider the expression

$$
s+a-\sqrt{s^{2}+2 s a} .
$$

This function is equal to the shifted version $s \rightarrow s+a$ of

$$
s-\sqrt{s^{2}-a^{2}},
$$

which is equal to

$$
s-\sqrt{s^{2}-a^{2}}=\frac{a^{2}}{s+\sqrt{s^{2}-a^{2}}}
$$

Now we note the following integral representation ( see pg 326 line 8 of [45]):

$$
\int_{0}^{\infty} \frac{x d x}{\left[x^{2}+2 s x+s^{2}-a^{2}\right]^{3 / 2}}=\frac{1}{s+\sqrt{s^{2}-a^{2}}}
$$

This function is equal to a Laplace transform (for all Laplace transforms used in this paper see [59])

$$
\begin{aligned}
\frac{1}{\left[x^{2}+2 s x+s^{2}-a^{2}\right]^{3 / 2}} & =\frac{1}{\left[(x+s)^{2}-a^{2}\right]^{3 / 2}} \\
& =\int_{0}^{\infty} d t e^{-(s+x) t} \frac{\sqrt{\pi}}{\Gamma(3 / 2)}\left(\frac{t}{2 a}\right) I_{1}(a t) .
\end{aligned}
$$

We simplify the numerical parts and again shift $s$ to $s+a$ to finally write

$$
\int_{0}^{\infty} d t \int_{0}^{\infty} x d x e^{-(s+a+x) t}\left(\frac{t}{a}\right) I_{1}(a t)=\int_{0}^{\infty} \frac{d t}{a t} I_{1}(a t) e^{-a t} e^{-s t} .
$$

In the original sums we recognize now $\sum_{\sigma} e^{-\epsilon_{\sigma} t}$ as integral of the heat kernel, which comes from the Laplace transform variable,

$$
\int_{0}^{\infty} d t \int_{0}^{\infty} x d x e^{-(s+a+x) t}\left(\frac{t}{a}\right) I_{1}(a t)=\int_{0}^{\infty} \frac{d t}{a t} I_{1}(a t) e^{-a t} \operatorname{Tr} e^{\Delta t}
$$


Let us see the convergence properties, since $\operatorname{Tr} e^{\Delta t}=O\left(t^{3 / 2}\right)$, and $I_{1}(t) \sim$ $e^{t} / \sqrt{t}$, there is no divergence as $t \rightarrow \infty$. However, as $t \rightarrow 0^{+}$which corresponds to the ultraviolet properties, we have $I_{1}(t) \sim t / 2$ we get

$$
\frac{1}{a t} I_{1}(a t) e^{-a t} \operatorname{Tr} e^{\Delta t} \sim \frac{1}{a t} \frac{a t}{2} \frac{1}{t^{3 / 2}} \text { as } t \rightarrow 0^{+} .
$$

which diverges, yet if we subtract from this expression $1 / 2$ we have

$$
\left(\frac{1}{a t} I_{1}(a t) e^{-a t}-\frac{1}{2}\right) \operatorname{Tr} e^{\Delta t} \sim \frac{1}{a t} \frac{a t}{2}(a t) \frac{1}{t^{3 / 2}} \text { as } t \rightarrow 0^{+} .
$$

which becomes convergent. The subtracted term, with the overall $a^{2}$ term being inserted back again, is indeed

$$
-\frac{a^{2}}{2} \operatorname{Tr} e^{\Delta t}=-\frac{a^{2}}{2} \int_{0}^{\infty} d t \sum_{\sigma} e^{-\epsilon_{\sigma} t}=-\sum_{\sigma} \frac{a^{2}}{2 \epsilon_{\sigma}} .
$$

Note that the subtraction does not lead to an infrared divergence, i.e. an ultraviolet divergence in the $t$ variable, thanks to $t^{-3 / 2}$ behavior of the heat kernel.

Rewriting the ground state energy by moving the chemical potential part to the right hand side we get

$$
E_{g}=\frac{u_{0} n_{0}^{2} V}{2}-\frac{a^{2} V}{2} \int_{0}^{\infty} d t\left(\frac{1}{a t} I_{1}(a t) e^{-a t}-\frac{1}{2}\right) \frac{1}{V} \operatorname{Tr} e^{\Delta t}
$$

here $a=u_{0} n_{0}$. Note that we can scale $a$ in the integrals to get

$$
E_{g}=\frac{u_{0} n_{0}^{2} V}{2}-\frac{a V}{2} \int_{0}^{\infty} d t\left(\frac{1}{t} I_{1}(t) e^{-t}-\frac{1}{2}\right) \frac{1}{V} \operatorname{Tr} e^{\Delta t / a}
$$

Let us recall the formula 3.534 from [45];

$$
\frac{I_{1}(t)}{t}=\frac{2}{\pi} \int_{0}^{1} d x \sqrt{1-x^{2}} \cosh (t x)
$$

moreover we have

$$
\int_{0}^{1} d x \sqrt{1-x^{2}}=\frac{\pi}{4}
$$

As a result we rewrite the ground state energy as

$$
E_{g}=\frac{u_{0} n_{0}^{2} V}{2}-\frac{a V}{\pi} \int_{0}^{\infty} d t \int_{0}^{1} d x \sqrt{1-x^{2}}\left(\cosh (t x) e^{-t}-1\right) \frac{1}{V} \operatorname{Tr} e^{\Delta t / a}
$$


or reorganizing this as

$$
E_{g}=\frac{u_{0} n_{0}^{2} V}{2}+\frac{a V}{2 \pi} \int_{0}^{\infty} d t \int_{0}^{1} d x F(t, x) \frac{1}{V} \operatorname{Tr} e^{\Delta t / a}
$$

where

$$
F(t, x)=\sqrt{1-x^{2}}(\underbrace{1-e^{-t(1-x)}}_{>0}+\underbrace{1-e^{-t(1+x)}}_{>0})>0 \text { for } 0 \leq x \leq 1 .
$$

This shows that the part multiplying the heat kernel is strictly positive, hence we may apply upper and lower bounds for the heat kernel to find estimates. Nevertheless we have a new version of the formula derived by Lee and Yang [3] in the flat space, extended now to a manifold, for the ground state energy.

How can we test this in the flat space? Let us write down the heat kernel in the flat space into the formula assuming $V \rightarrow \infty$ for simplicity and putting back again the physical values for the energy eigenvalues, i.e. heat kernel corrected with a factor of $\hbar^{2} / 2 m$ :

$$
E_{g}=\frac{u_{0} n_{0}^{2} V}{2}+\frac{a V}{2 \pi} \int_{0}^{\infty} d t \int_{0}^{1} d x F(t, x) \frac{a^{3 / 2} 2^{3 / 2} m^{3 / 2}}{\hbar^{3}(4 \pi t)^{3 / 2}}
$$

We now recall that

$$
\int_{0}^{\infty} \frac{d t}{t^{3 / 2}}\left(1-e^{-t(1 \pm x)}\right)=2 \sqrt{\pi}(1 \pm x)^{1 / 2}
$$

Thus we get

$$
\begin{aligned}
E_{g}=\frac{u_{0} n_{0}^{2} V}{2} & {\left[1+\frac{2^{5 / 2}(\pi)^{1 / 2}}{\pi n_{0}} \frac{a^{3 / 2} m^{3 / 2}}{\hbar^{3}(4 \pi)^{3 / 2}} \int_{0}^{1} d x \sqrt{1-x^{2}}\right.} \\
& \left.\left((1+x)^{1 / 2}+(1-x)^{1 / 2}\right)\right]
\end{aligned}
$$

The integral can be written as

$$
\int_{0}^{1} d x\left((1-x)^{1 / 2}(1+x)+(1+x)^{1 / 2}(1-x)\right)
$$

by the formula 3.214 in [45] and is equal to $2^{3 / 2+2-1} B(3 / 2,2)(B$ is the beta function).

$$
E_{g}=\frac{u_{0} n_{0} N}{2}\left[1+\frac{2^{5 / 2}}{\pi^{1 / 2} n_{0}}\left(2^{5 / 2} B(3 / 2,2)\right) \frac{a^{3 / 2} m^{3 / 2}}{\hbar^{3}(4 \pi)^{3 / 2}}\right]
$$


Now note that $u_{0}=4 \pi \sigma \hbar^{2} / m$. Hence in terms of the scattering cross section, the energy per particle becomes,

$$
\frac{E_{g}}{N}=\frac{2 \pi \hbar^{2} \sigma n_{0}}{m}\left[1+\frac{2^{5 / 2}}{\pi^{1 / 2}}\left(2^{5 / 2} B(3 / 2,2)\right) n_{0}^{1 / 2} \sigma^{3 / 2}\right] .
$$

We have $B(3 / 2,2)=4 / 15$, thus

$$
\frac{E_{g}}{N}=\frac{2 \pi \hbar^{2} \sigma n_{0}}{m}\left[1+\frac{128}{15 \pi^{1 / 2}}\left(n_{0} \sigma^{3}\right)^{1 / 2}\right] .
$$

the well-known result of Lee and Yang is recovered.

The expression we have found opens up two different directions: first is that we may find upper and lower bound estimates for the ground state energy using the known bounds of the heat kernel. We will now present an upper bound in the thermodynamic limit for the ground state energy of the interacting system. To this effect we use formula (11) for the upper bound of the trace of the heat kernel, place this into the expression for the ground state energy, replace the upper limit of $t$-integral by infinity and estimate the remainder term; this results in

$$
E_{g} \leq C(3) E_{g}^{f l a t}+C(3) \frac{a^{2}}{2 \pi} \int_{D_{M}^{2}}^{\infty} d t \int_{0}^{1} d x F_{a}(t, x)\left[\frac{D_{M}^{3}}{t^{3 / 2}}+1\right]
$$

where we use the natural units $\hbar=1,2 m=1$ again for simplicity, and $C(3)$ is a constant which depends on the dimension $d=3$ only, $D_{M}$ is the diameter of the box as discussed before. We have

$$
F_{a}(t, x)=\sqrt{1-x^{2}}\left[1-e^{-a t(x-1)}+1-e^{-a t(x+1)}\right]
$$

Note that we have

$$
F_{a}(t, x)<2 \sqrt{1-x^{2}}\left[1-e^{-2 a t}\right]
$$

We now argue that the last term is a lower order correction, as we take the limit $V \rightarrow \infty$. It is easy to see that the constant term is not of much importance, so we focus on the $1 / t^{3 / 2}$ term. This term can be written as,

$$
\begin{aligned}
C(3) \frac{a^{2} D_{M}^{3}}{\pi} \int_{D_{M}^{2}}^{\infty} d t \int_{0}^{1} d x & \left(1-x^{2}\right)^{1 / 2} \int_{0}^{2 a} d \eta e^{-\eta t} t \frac{1}{t^{3 / 2}} \\
= & C(3) a^{2} D_{M}^{3} \int_{0}^{2 a} d \eta \int_{D_{M}}^{\infty} e^{-\eta u^{2}} d u
\end{aligned}
$$

We now observe that

$$
\int_{0}^{2 a} d \eta \int_{D_{M}}^{\infty} e^{-\eta u^{2}} d u<\int_{0}^{2 a} d \eta e^{-\eta D_{M}^{2}} \int_{0}^{\infty} e^{-\eta u^{2}} d u=\int_{0}^{2 a} d \eta \frac{e^{-\eta D_{M}^{2}}}{\sqrt{\eta}}
$$


As a result we see that this term is less than

$$
C(3) a^{2} D_{M}^{3} \int_{0}^{2 a} d \eta \frac{e^{-\eta D_{M}^{2}}}{\sqrt{\eta}}<C(3) \frac{a^{2} D_{M}^{3} \sqrt{\pi}}{2} \frac{1}{D_{M}}
$$

which goes to zero for $E_{g} / N$ when we take the limit $V \rightarrow \infty$, hence it is a lower order correction when we assume $V \approx O\left(D_{M}^{3}\right)$.

As a second alternative, we may write down the finite volume version of the heat kernel, and find the corrections which may be coming from the boundary effects. A first guess would be to apply an asymptotic expansion for the short time behaviour of the heat kernel. Finite size effects for the condensation of the free gas from this perspective is presented in [46], following the idea in this work we will obtain finite size effects of the weakly interacting condensate. Let us digress a bit on the asymptotics of the Euclidean Domains which denote as $\Omega$. In three dimensions the precise asymptotics for the Neumann problem is given by [47]

$$
\operatorname{Tr} e^{\Delta t}=\frac{V(\Omega)}{(4 \pi t)^{3 / 2}}+\frac{A(\Omega)}{16 \pi t}+\frac{1}{12 \pi^{3 / 2} t^{1 / 2}} \int_{\partial \Omega} H(\omega) d \omega+\ldots \quad \text { as } t \rightarrow 0^{+}
$$

where $A(\Omega)$ and $H(\omega)$ refer to the area and mean curvature of the surface respectively. To use this in our expression we rewrite it as,

$$
\begin{aligned}
E_{g} \approx & \frac{u_{0} n_{0}^{2} V(\Omega)}{2}+\frac{a V(\Omega)}{2 \pi} \int_{0}^{\infty} d t \int_{0}^{1} d x\left[F(t, x) \frac{a^{3 / 2}}{(4 \pi t)^{3 / 2}}\right] \\
+ & \frac{a}{2 \pi} \int_{0}^{1} d t \int_{0}^{1} d x F(t, x)\left(\frac{A(\Omega) a}{16 \pi t}+\frac{a^{1 / 2}}{12 \pi^{3 / 2} t^{1 / 2}} \int_{\partial \Omega} H(\omega) d \omega+\ldots\right) \\
& +\frac{a V(\Omega)}{2 \pi} \int_{1}^{\infty} d t \int_{0}^{1} d x F(t, x)\left(\frac{1}{V(\Omega)} \operatorname{Tr} e^{\Delta t / a}-\frac{a^{3 / 2}}{(4 \pi t)^{3 / 2}}\right)
\end{aligned}
$$

Now we claim that the last term of this expression can be made smaller than the others, hence of less significance. We leave this question to a subsequent publication. Retaining the terms in the above expansion except the last part, we find finite size corrections to the interacting Bose-Einstein condensate.

Finally we can also calculate the chemical potential, by taking the derivative with respect to the particle number. This gives us,

$$
\begin{aligned}
& \frac{\partial E_{g}}{\partial N}=u_{0} n_{0}+u_{0} n_{0}^{2} \pi \int_{0}^{\infty} d t \int_{0}^{1} d x \sqrt{1-x^{2}} F(t, x) \frac{1}{V} \operatorname{Tr} e^{\Delta t} \\
& +u_{0}^{2} n_{0} \int_{0}^{\infty} d t \int_{0}^{1} d x a \sqrt{1-x^{2}}\left((1-x) e^{-a t(1-x)}+(1+x) e^{-a t(1+x)}\right) \frac{1}{V} \operatorname{Tr} e^{\Delta t} .
\end{aligned}
$$




\section{Heat Kernel Analysis of the Depletion of the Condensate}

We will now study the depletion coefficient in $3 d$ and $2 d$, first at zero then at finite temperature. The number density of excited states

$$
n_{e}=\frac{1}{V} \sum_{\sigma \neq 0}\left\langle a_{\sigma}^{\dagger} a_{\sigma}\right\rangle
$$

is expressed in terms of quasi-particle states as

$$
\begin{aligned}
n_{e} & =\frac{1}{V} \sum_{\sigma \neq 0}\left[\sinh ^{2} \xi_{\sigma}+\cosh 2 \xi_{\sigma}\left\langle b_{\sigma}^{\dagger} b_{\sigma}\right\rangle+\frac{1}{2} \sinh 2 \xi_{\sigma}\left\langle b_{\sigma}^{\dagger 2}+b_{\sigma}^{2}\right\rangle\right] \\
& =\frac{1}{V} \sum_{\sigma \neq 0}\left[\cosh 2 \xi_{\sigma}\left(\frac{1}{2}+\left\langle b_{\sigma}^{\dagger} b_{\sigma}\right\rangle\right)-\frac{1}{2}\right] \\
& =\frac{1}{V} \sum_{\sigma \neq 0}\left[\frac{1}{2} \cosh 2 \xi_{\sigma} \operatorname{coth} \frac{\beta \omega_{\sigma}}{2}-\frac{1}{2}\right] \\
& =\frac{1}{2 V} \sum_{\sigma \neq 0}\left[\frac{\lambda_{\sigma}}{\omega_{\sigma}} \operatorname{coth} \frac{\beta \omega_{\sigma}}{2}-1\right] .
\end{aligned}
$$

The zero temperature limit is

$$
n_{e}=\frac{1}{2 V} \sum_{\sigma \neq 0}\left[\frac{\lambda_{\sigma}}{\sqrt{\lambda_{\sigma}^{2}-\left(u_{0} n_{0}\right)^{2}}}-1\right] .
$$

Now noting the Laplace transform

$$
\frac{1}{\sqrt{\lambda_{\sigma}^{2}-\left(u_{0} n_{0}\right)^{2}}}=\int_{0}^{\infty} d t e^{-\lambda_{\sigma} t} I_{0}\left(u_{0} n_{0} t\right)
$$

where $I_{\nu}$ is the modified Bessel function of order $\nu$, we get

$$
\begin{aligned}
\frac{\lambda_{\sigma}}{\sqrt{\lambda_{\sigma}^{2}-\left(u_{0} n_{0}\right)^{2}}} & =\int_{0}^{\infty} d t\left[-\frac{d}{d t} e^{-\lambda_{\sigma} t}\right] I_{0}\left(u_{0} n_{0} t\right) \\
& =1+u_{0} n_{0} \int_{0}^{\infty} d t e^{-\lambda_{\sigma} t} I_{1}\left(u_{0} n_{0} t\right) .
\end{aligned}
$$


Thus

$$
\begin{aligned}
n_{e} & =\frac{u_{0} n_{0}}{2 V} \int_{0}^{\infty} d t\left[\sum_{\sigma \neq 0} e^{-\epsilon_{\sigma} t}\right] e^{-u_{0} n_{0} t} I_{1}\left(u_{0} n_{0} t\right) \\
& =\frac{u_{0} n_{0}}{2} \int_{0}^{\infty} d t \frac{1}{V} \operatorname{Tr}^{\prime} e^{-h t} e^{-u_{0} n_{0} t} I_{1}\left(u_{0} n_{0} t\right) .
\end{aligned}
$$

Finite Volume: At finite $V$, we can use either (17) or the simpler observation

$$
\operatorname{Tr}^{\prime} e^{-h t}=\sum_{\sigma=1}^{\infty} e^{-t \epsilon_{\sigma}} \sim e^{-t \epsilon_{1}}
$$

together with

$$
e^{-u_{0} n_{0} t} I_{1}\left(u_{0} n_{0} t\right) \sim \frac{1}{\sqrt{2 \pi u_{0} n_{0} t}} \text { as } t \rightarrow \infty,
$$

and conclude that the upper limit of (125) is finite. As for the lower limit of integration, we combine the short time asymptotic of the heat kernel

$$
\operatorname{Tr}^{\prime} e^{-h t} \sim-1+\frac{V}{(4 \pi t)^{d / 2}}, \quad \text { as } \quad t \rightarrow 0
$$

with

$$
e^{-u_{0} n_{0} t} I_{1}\left(u_{0} n_{0} t\right) \sim \frac{u_{0} n_{0} t}{2} \text { as } t \rightarrow 0,
$$

to conclude that the integral is convergent for $d=3$ and $d=2$.

Thermodynamic Limit: Using (16) in the limit $V, D_{M} \rightarrow \infty, D_{M}^{d} / V \rightarrow A$ we get

$$
n_{e} \leq \frac{u_{0} n_{0}}{2} \frac{A}{C^{d / 2}} \Gamma\left(\frac{d}{2}+1\right) \int_{0}^{\infty} d t \frac{1}{t^{d / 2}} e^{-u_{0} n_{0} t} I_{1}\left(u_{0} n_{0} t\right) .
$$

The integral is convergent for $d=3$.

On the other hand

$$
n_{e} \geq \frac{u_{0} n_{0}}{2} \frac{1}{B^{d / 2}} \frac{d}{2} \int_{0}^{\infty} d t \Gamma\left(\frac{d}{2}, \frac{t B}{V^{2 / d}}\right) \frac{1}{t^{d / 2}} e^{-u_{0} n_{0} t} I_{1}\left(u_{0} n_{0} t\right) .
$$

Again the integral is convergent for $d=3$ and $d=2$. Moreover, the integrand is a positive, increasing function of $V$ and by the monotone convergence theorem the limit $V \rightarrow \infty$ gives

$$
n_{e} \geq \frac{u_{0} n_{0}}{2} \frac{1}{B^{d / 2}} \Gamma\left(\frac{d}{2}+1\right) \int_{0}^{\infty} d t \frac{1}{t^{d / 2}} e^{-u_{0} n_{0} t} I_{1}\left(u_{0} n_{0} t\right) .
$$


Finally, upon the change of variable $s=u_{0} n_{0} t$ we see that the bounds scale as $\left(u_{0} n_{0}\right)^{d / 2}$

$$
\left(u_{0} n_{0}\right)^{d / 2} m \gamma_{d} \leq n_{e} \leq\left(u_{0} n_{0}\right)^{d / 2} M \gamma_{d}
$$

where

$$
m=\frac{1}{2} \frac{1}{B^{d / 2}} \Gamma\left(\frac{d}{2}+1\right), \quad M=\frac{1}{2} \frac{A}{C^{d / 2}} \Gamma\left(\frac{d}{2}+1\right)
$$

and

$$
\gamma_{d}=\int_{0}^{\infty} \frac{d s}{s^{d / 2}} e^{-s} I_{1}(s) .
$$

Thus we get, as in the flat case,

$$
\frac{n_{e}}{n_{0}}=O\left(u_{0}^{d / 2} n_{0}^{d / 2-1}\right) .
$$

The smallness of the parameter $u_{0}^{d / 2} n_{0}^{d / 2-1}$ can now be used as a criterion for the validity of the Gross-Pitaevskii equation (see e.g. [15]).

\section{Depletion of the Condensate at Finite Tem- perature}

In order to analyze the depletion of the condensate at finite temperatures we expand $N_{e}$ in terms of exponentials

$$
N_{e}(T)=\frac{1}{2} \sum_{\sigma \neq 0}\left[\frac{\lambda_{\sigma}}{\omega_{\sigma}}-1+2 \sum_{k=1}^{\infty} \frac{\lambda_{\sigma}}{\omega_{\sigma}} e^{-k \beta \omega_{\sigma}}\right] .
$$

Noticing the Laplace transform,

$$
\frac{e^{-k \beta \omega_{\sigma}}}{\omega_{\sigma}}=\frac{e^{-k \beta \sqrt{\lambda_{\sigma}^{2}-\left(u_{0} n_{0}\right)^{2}}}}{\sqrt{\lambda_{\sigma}^{2}-\left(u_{0} n_{0}\right)^{2}}}=\int_{k \beta}^{\infty} d t e^{-\lambda_{\sigma} t} I_{0}\left(u_{0} n_{0} \sqrt{t^{2}-k^{2} \beta^{2}}\right),
$$

we find

$$
\begin{aligned}
\frac{\lambda_{\sigma} e^{-k \beta \omega_{\sigma}}}{\omega_{\sigma}} & =e^{-\lambda_{\sigma} k \beta}+u_{0} n_{0} \int_{k \beta}^{\infty} d t e^{-\lambda_{\sigma} t} \frac{t I_{1}\left(u_{0} n_{0} \sqrt{t^{2}-k^{2} \beta^{2}}\right)}{\sqrt{t^{2}-k^{2} \beta^{2}}} \\
& =e^{-\lambda_{\sigma} k \beta}+u_{0} n_{0} \int_{0}^{\infty} d t e^{-\lambda_{\sigma} \sqrt{t^{2}+k^{2} \beta^{2}}} I_{1}\left(u_{0} n_{0} t\right) .
\end{aligned}
$$

Thus, we arrive at

$$
n_{e}(T)=n_{e}(0)+\tilde{n}_{e}(T),
$$


where

$$
\begin{aligned}
\tilde{n}_{e}(T)= & \frac{1}{V} \sum_{\sigma \neq 0} \sum_{k=1}^{\infty}\left[e^{-\lambda_{\sigma} k \beta}+u_{0} n_{0} \int_{0}^{\infty} d t e^{-\lambda_{\sigma} \sqrt{t^{2}+k^{2} \beta^{2}}} I_{1}\left(u_{0} n_{0} t\right)\right] \\
= & \sum_{k=1}^{\infty}\left[\left(\frac{1}{V} \operatorname{Tr}^{\prime} e^{k \beta \Delta}\right) e^{-k \beta u_{0} n_{0}}+\right. \\
& \left.u_{0} n_{0} \int_{0}^{\infty} d t\left(\frac{1}{V} \operatorname{Tr}^{\prime} e^{\Delta \sqrt{t^{2}+k^{2} \beta^{2}}}\right) e^{-u_{0} n_{0} \sqrt{t^{2}+k^{2} \beta^{2}}} I_{1}\left(u_{0} n_{0} t\right)\right] .
\end{aligned}
$$

We will now analyze this expression in the thermodynamic limit in $3 d$. Using the heat kernel upper bound (16) in the limit $V \rightarrow \infty$ we get

$$
\begin{aligned}
\tilde{n}_{e}(T) \leq & C_{1} \sum_{k=1}^{\infty}\left[\frac{1}{(k \beta)^{3 / 2}} e^{-k \beta u_{0} n_{0}}+\right. \\
& \left.u_{0} n_{0} \int_{0}^{\infty} d t \frac{1}{\left(t^{2}+k^{2} \beta^{2}\right)^{3 / 4}} e^{-u_{0} n_{0} \sqrt{t^{2}+k^{2} \beta^{2}}} I_{1}\left(u_{0} n_{0} t\right)\right] .
\end{aligned}
$$

Here

$$
C_{1}=\frac{A}{C^{3 / 2}} \Gamma\left(\frac{5}{2}\right)
$$

We will estimate each term separately, the first expression is bounded by

$$
C_{1} \sum_{k=1}^{\infty} \frac{1}{(k \beta)^{3 / 2}}+C_{1} \int_{0}^{\infty} \frac{d k}{(\beta k)^{3 / 2}}\left(1-e^{-k \beta u_{0} n_{0}}\right),
$$

which is equal to

$$
C_{1} \sum_{k=1}^{\infty} \frac{1}{(k \beta)^{3 / 2}}+C_{1} \frac{\left(u_{0} n_{0}\right)^{1 / 2}}{\beta} .
$$

The second part is somewhat more subtle, we first apply the subordination identity for the exponent and find that the second becomes,

$$
C_{2} u_{0} n_{0} \int_{0}^{\infty} d t \sum_{k=1}^{\infty} \frac{1}{\left(t^{2}+k^{2} \beta^{2}\right)^{3 / 4}} \int_{0}^{\infty} \frac{d s}{s^{3 / 2}} e^{-\frac{1}{4 s}-s\left(u_{0} n_{0}\right)^{2}\left(t^{2}+k^{2} \beta^{2}\right)} I_{1}\left(u_{0} n_{0} t\right) .
$$


Next we estimate the summation, again the terms of the sum are monotonically decreasing as the summand increases, hence the integral gives an upper bound which we estimate separately;

$$
\begin{aligned}
& \sum_{k=1}^{\infty} \frac{1}{\left(t^{2}+k^{2} \beta^{2}\right)^{3 / 4}} e^{-\left(u_{0} n_{0}\right)^{2} s k^{2} \beta^{2}}<\int_{0}^{\infty} \frac{d k}{\left(t^{2}+k^{2} \beta^{2}\right)^{3 / 4}} e^{-\left(u_{0} n_{0}\right)^{2} s k^{2} \beta^{2}} \\
< & {\left[\int_{0}^{\infty} \frac{d k}{\left(t^{2}+k^{2} \beta^{2}\right)^{3 / 2}}\right]^{1 / 2}\left[\int_{0}^{\infty} d k e^{-2 s\left(u_{o} n_{0}\right)^{2} k^{2} \beta^{2}}\right]^{1 / 2} } \\
< & C_{3} \frac{1}{s^{1 / 4}\left(u_{0} n_{0}\right)^{1 / 2} \beta^{1 / 2}} \frac{1}{\beta^{1 / 2} t} .
\end{aligned}
$$

Note that in the first integral we scale the variable $k$ with $t \beta^{-1}$ and the second integral by $\left(\sqrt{s} \beta u_{0} n_{0}\right)^{-1}$. We may place this estimate now to find the upper bound,

$$
\frac{C_{4}\left(u_{0} n_{0}\right)^{1 / 2}}{\beta} \int_{0}^{\infty} \frac{d t}{t} \int_{0}^{\infty} \frac{d s}{s^{1+3 / 4}} e^{-\frac{1}{4 s}-s\left(u_{0} n_{0}\right)^{2} t^{2}} I_{1}\left(u_{0} n_{0} t\right) .
$$

We recognize the modified bessel function, to rewrite this expression as,

$$
\frac{C_{5}\left(u_{0} n_{0}\right)^{1 / 2}}{\beta} \int_{0}^{\infty} \frac{d t}{t}\left(u_{0} n_{0} t\right)^{3 / 4} K_{3 / 4}\left(u_{0} n_{0} t\right) I_{1}\left(u_{0} n_{0} t\right) .
$$

Note that in the integral $\left(u_{0} n_{0}\right)$ completely scales out. The integral is of the type given in Prudnikov et al. [57] formula 2.16.28.3.

$$
\int_{0}^{\infty} d x x^{\rho-1} K_{\mu}(x) I_{\nu}(x)=\frac{2^{\rho-1} \Gamma\left(\frac{1}{2}(\rho+\nu+\mu)\right) \Gamma\left(\frac{1}{2}(\rho+\nu-\mu)\right) \Gamma(1-\rho)}{\Gamma\left(1+\frac{1}{2}(-\rho+\nu+\mu)\right) \Gamma\left(1+\frac{1}{2}(-\rho+\nu-\mu)\right)} .
$$

for $|\mu|-\nu<\rho<1$. Hence we find that

$$
\tilde{n}_{e}(T)<C_{1} \sum_{k=1}^{\infty} \frac{1}{(k \beta)^{3 / 2}}+C_{6} \frac{\left(u_{0} n_{0}\right)^{1 / 2}}{\beta},
$$

the last piece of which will go to zero as $u_{0} \rightarrow 0^{+}$and moreover the full expression will go to zero as $\beta \rightarrow \infty$.

Next, we will show that the Bogoliubov approximation at finite temperature is actually inconsistent in $2 d$, in accordance with the HohenbergMermin-Wagner teorem [58], [36], rigorously established in spin systems or interacting bosons in flat spaces. To see this, we will use lower bounds on the heat kernel (16) in the limit $V \rightarrow \infty$. We will further estimate sums of 
monotonically decreasing expressions from below by integrating them from 1 to infinity. As a result we see that $\tilde{n}_{e}(T)$ is larger than

$\frac{1}{\beta} \int_{1}^{\infty} \frac{d k}{k} e^{-\left(u_{0} n_{0}\right) k \beta}+u_{0} n_{0} \int_{0}^{\infty} d t \int_{1}^{\infty} \frac{d k}{\sqrt{t^{2}+k^{2} \beta^{2}}} e^{-u_{0} n_{0} \sqrt{t^{2}+k^{2} \beta^{2}}} I_{1}\left(u_{0} n_{0} t\right)$.

Note that we may shift $k$ to $k+1$ and replace all $k^{2}+2 k+1$ 's by $3 k^{2}+1$ since $k^{2}+2 k+1<3 k^{2}+1$ for $k \geq 0$. After scaling $k$ to $k \sqrt{t^{2}+\beta^{2}} / \sqrt{3} \beta$ we find that $\tilde{n}_{e}(T)$ is larger than

$\frac{1}{\beta} \int_{1}^{\infty} \frac{d k}{k} e^{-\left(u_{0} n_{0}\right) k \beta}+\frac{u_{0} n_{0}}{\sqrt{3} \beta} \int_{0}^{\infty} d t \int_{0}^{\infty} \frac{d k}{\sqrt{1+k^{2}}} e^{-u_{0} n_{0} \sqrt{t^{2}+\beta^{2}} \sqrt{1+k^{2}}} I_{1}\left(u_{0} n_{0} t\right)(1$.

We notice that

$$
\int_{0}^{\infty} \frac{d k e^{-a \sqrt{1+k^{2}}}}{\sqrt{1+k^{2}}}=K_{0}(a),
$$

hence the lower bound becomes,

$$
\frac{1}{\beta} \int_{1}^{\infty} \frac{d k}{k} e^{-\left(u_{0} n_{0}\right) k \beta}+\frac{u_{0} n_{0}}{\sqrt{3} \beta} \int_{0}^{\infty} d t K_{0}\left(u_{0} n_{0} \sqrt{t^{2}+\beta^{2}}\right) I_{1}\left(u_{0} n_{0} t\right)<\tilde{n}_{e}(T)(
$$

Nevertheless the integral of Bessel functions is ultraviolet divergent-which reflects the infrared behavior of the theory in the heat kernel approach-as a result of the asymptotics of the bessel functions,

$$
K_{0}(x) \sim \frac{e^{-x}}{\sqrt{x}} \quad \text { and } \quad I_{1}(x) \sim \frac{e^{x}}{\sqrt{x}} \quad \text { as } \quad x \rightarrow \infty .
$$

This contradiction forces $n_{0}=0$ to be the only consistent choice.

\section{Acknowledgement}

This work is supported by Boğaziçi University BAP Project No. 6942. We would like to thank Dieter Van den Bleeken and Yusuf Gül for useful discussions.

\section{Appendix A}

Here we will show how the upper bound (12) for the eigenvalues follows from the upper estimate for the trace of the heat kernel given in (11) [34], [56], and 
how one gets the long time behavior (17) from the self-reproducing property of the heat kernel.

Starting from the simple observation

$$
(\sigma+1) e^{-\epsilon_{\sigma} t} \leq \operatorname{Tr} e^{\Delta t} \leq \tilde{C}(d) g(t),
$$

and using the upper bound

$$
\operatorname{Tr} e^{\Delta t} \leq \tilde{C}(d) g(t)
$$

we get

$$
(\sigma+1) \leq e^{\epsilon_{\sigma} t} \tilde{C}(d) g(t)
$$

for all positive $t$. Minimizing the right hand side we get

$$
\epsilon_{\sigma} g\left(t_{0}\right)+g^{\prime}\left(t_{0}\right)=0 .
$$

Since $g^{\prime}(t)=0$ for $\sqrt{t}>D_{M}$, we see that $\sqrt{t_{0}}<D_{M}$. Then we get

$$
t_{0}=\frac{d}{2 \epsilon_{\sigma}} \text {. }
$$

Plugging this into (159) we get the desired bound

$$
\epsilon_{\sigma} \geq \frac{C(d)}{D_{M}^{2}}(\sigma+1)^{2 / d} \geq \frac{C(d)}{D_{M}^{2}} \sigma^{2 / d} .
$$

Let $K_{t}(x, y)=\left\langle x\left|e^{\Delta t}\right| y\right\rangle$ be the heat kernel for the Neumann problem on $M$. Clearly $K_{t}(x, y)$ is a self-reproducing kernel. It is convenient to define

$$
\bar{K}_{t}(x, y)=K_{t}(x, y)-\frac{1}{V} .
$$

Since $V^{-1 / 2}$ is the eigenfunction of the Laplacian with zero eigenvalue we have

$$
\frac{1}{\sqrt{V}}=\int_{M} d_{g} \mu(y) K_{t}(x, y) \frac{1}{\sqrt{V}} .
$$

Using this it is easy to see that $\bar{K}_{t}$ is also self-reproducing

$$
\bar{K}_{t_{1}+t_{2}}(x, y)=\int_{M} d_{g} \mu(z) \bar{K}_{t_{1}}(x, z) \bar{K}_{t_{2}}(z, y) .
$$

Now note that [54]

$$
\begin{aligned}
\frac{\partial}{\partial t} \bar{K}_{t}(x, x) & =\frac{\partial}{\partial t} \int_{M} d_{g} \mu(z) \bar{K}_{t / 2}^{2}(x, z) \\
& =-\int_{M} d_{g} \mu(z) \bar{K}_{t / 2}(x, z) h \bar{K}_{t / 2}(x, z) \\
& \leq-\epsilon_{1} \int_{M} d_{g} \mu(z) \bar{K}_{t / 2}^{2}(x, z)=-\epsilon_{1} \bar{K}_{t}(x, x) .
\end{aligned}
$$


In the last line the variational inequality is used (see e.g. [55]). Integrating this inequality one finds that for $t \geq t_{0}$

$$
\bar{K}_{t}(x, x) \leq \bar{K}_{t_{0}}(x, x) e^{-\epsilon_{1}\left(t-t_{0}\right)} .
$$

Fixing the value of $t_{0}$ we see that the diagonal elements of $\bar{K}_{t}$ decay exponentially in time. Integrating over $x$ we get

$$
\operatorname{Tr}^{\prime} e^{\Delta t} \leq\left(\operatorname{Tr}^{\prime} e^{\Delta t_{0}}\right) e^{-\nu_{1}\left(t-t_{0}\right)} .
$$

\section{Appendix B}

Let $\{|z\rangle z \in N\}$ be an over-complete set labeled by the points of a manifold $N$ with measure $d \mu(z)$. The lower symbol $A_{L}(z)$ of an operator $A$ is the expectation value

$$
A_{L}(z)=\langle z|A| z\rangle
$$

On the other hand, if there exist a function $A_{U}(z)$ on $N$ such that

$$
A=\int d \mu(z) A_{U}(z)|z\rangle\langle z|
$$

then $A_{U}(z)$ is called the upper symbol of $A$. Here the equality is in the weak sense.

Let

$$
|z\rangle=e^{-\frac{\left|z^{2}\right|}{2}+z a_{0}^{\dagger}}|0\rangle, \quad z \in \mathbf{C},
$$

be the standard coherent states for the annihilation operator $a_{0}$. This is an over-complete set relative to the measure

$$
d \mu(z)=\frac{d z d z^{*}}{\pi}
$$

The following list of the symbols of various combinations of creation and annihilation operators is useful in calculating the lower and upper symbols of the Hamiltonian.

\begin{tabular}{|l|l|l|}
\hline$A$ & $A_{L}$ & $A_{U}$ \\
\hline$a_{0}$ & $z$ & $z$ \\
$a_{0}^{\dagger}$ & $z^{*}$ & $z^{*}$ \\
$a_{0}^{2}$ & $z^{2}$ & $z^{2}$ \\
$a_{0}^{\dagger 2}$ & $z^{* 2}$ & $z^{* 2}$ \\
$a_{0}^{\dagger} a_{0}$ & $|z|^{2}$ & $|z|^{2}-1$ \\
$a_{0}^{\dagger 2} a_{0}^{2}$ & $|z|^{4}$ & $|z|^{4}-4|z|^{2}+2$ \\
\hline
\end{tabular}


We assume our Hamiltonian includes the chemical potential

$$
H=H^{\prime}-\mu N
$$

where $H^{\prime}$ is given by (66). In the Hamiltonian $H$ we replace every monomial of the form $a_{0}^{\dagger n} a_{0}^{m}$ first by its lower and then by its upper symbol and thus obtain the two Hamiltonians $H_{L}\left(z, z^{*}\right)$ and $H_{U}\left(z, z^{*}\right)$. Let $Z_{L}(\beta, \mu)$ and $Z_{U}(\beta, \mu)$ be the corresponding grand canonical partition functions integrated over $z$,

$$
Z_{L, U}(\beta, \mu)=\int \frac{d z d z^{*}}{\pi} Z_{L, U}\left(\beta, \mu, z, z^{*}\right)
$$

where

$$
Z_{L, U}\left(\beta, \mu, z, z^{*}\right)=\operatorname{Tr}^{\prime} e^{-\beta H_{L, U}\left(z, z^{*}\right)} .
$$

Here $\operatorname{Tr}^{\prime}$ means that the trace is taken over the states with no excitations in the $f_{0}$ mode. Then we have the following inequalities

$$
Z_{L}(\beta, \mu) \leq Z(\beta, \mu) \leq Z_{U}(\beta, \mu)
$$

The first inequality is the Jensen's inequality and the second is the BerezinLieb inequality [49], [50], [51], [52], [53], [8]. These inequalities are valid on any manifold since geometry does not play any role whatsoever in their derivations.

Comparing $H_{L}$ and $H_{U}$ we see that

$$
\begin{aligned}
\delta & =H_{U}\left(z, z^{*}\right)-H_{L}\left(z, z^{*}\right)=\mu+\frac{u_{0}}{4 V}\left(-4|z|^{2}+2-4 \sum_{\sigma \neq 0} a_{\sigma}^{\dagger} a_{\sigma}\right) \\
& =\mu+\frac{u_{0}}{4 V}\left(2-4 N_{L}\right) .
\end{aligned}
$$

In deriving this we used the list of symbols given above and the fact that $f_{0}=V^{-1 / 2}$.

Thus we find

$$
\begin{aligned}
\operatorname{Tr}^{\prime} e^{-\beta H_{U}\left(z, z^{*}\right)} & =\operatorname{Tr}^{\prime} e^{-\beta\left(H_{L}\left(z, z^{*}\right)+\mu+\frac{u_{0}}{V}-\frac{2 u_{0}}{V} N_{L}\right)} \\
& =e^{-\beta\left(\mu+\frac{u_{0}}{V}\right)} \operatorname{Tr}^{\prime} e^{-\beta\left(H_{L}\left(z, z^{*}\right)-\frac{2 u_{0}}{V} N_{L}\right)}
\end{aligned}
$$

or

$$
Z_{U}(\mu, \beta)=e^{-\beta\left(\mu+\frac{u_{0}}{V}\right)} Z_{L}\left(\mu+\frac{2 u_{0}}{V}, \beta\right)
$$


So

$$
\begin{aligned}
\lim _{V \rightarrow \infty} \frac{1}{V} \ln Z_{U}(\mu, \beta) & =\lim _{V \rightarrow \infty} \frac{1}{V}\left[-\beta\left(\mu+\frac{u_{0}}{V}\right)\right]+\frac{1}{V} \ln Z_{L}\left(\mu+\frac{2 u_{0}}{V}, \beta\right) \\
& =\lim _{V \rightarrow \infty} \frac{1}{V} \ln Z_{L}(\mu, \beta) .
\end{aligned}
$$

Then from (177) we get the equality of the pressures in the thermodynamic limit

$$
\frac{1}{V} \ln Z_{U}(\mu, \beta)=\frac{1}{V} \ln Z(\mu, \beta)=\frac{1}{V} \ln Z_{L}(\mu, \beta) .
$$

Let $z_{0}$ be the value of $z$ for which $Z_{L}\left(\beta, \mu, z, z^{*}\right)$ is maximum. Then the integrals in the above expressions localize around $z_{0}$ and the following inequalities hold [8]

$$
\frac{1}{V} \ln Z_{L}\left(\mu, \beta, z_{0}, z_{0}^{*}\right) \leq \frac{1}{V} \ln Z(\mu, \beta) \leq \frac{1}{V} \ln Z_{U}\left(\mu, \beta, z_{0}, z_{0}^{*}\right)+O\left(\frac{\ln V}{V}\right) .
$$

Note that the usual choice $\mu=u_{0} n_{0}$ in the Bogoliubov theory is in accordance with this result. Again using (178), in the thermodynamic limit we obtain

$$
\frac{1}{V} \ln Z(\mu, \beta)=\frac{1}{V} \ln Z_{L}\left(\mu, \beta, z_{0}, z_{0}^{*}\right) .
$$

\section{References}

[1] N. N. Bogoliubov, J. Phys. USSR 11, 23 (1947).

[2] K. Huang, C. N. Yang, Phys. Rev. 105, 767 (1957).

[3] T. D. Lee, C. N. Yang, Phys. Rev. 105, 1119 (1957).

[4] T. D. Lee, K. Huang, C. N. Yang, Phys. Rev. 106, 1135, (1957).

[5] S. Gallot, D. Hulin, and J. Lafontaine, Riemannian Geometry Springer Verlag, 3rd edition, New York (2004).

[6] G. Wei, Manifolds with a lower Ricci bound, Surveys in Differential Geometry XI, edited by J. Cheeger and K. Grove, International Press, Massachusetts(2007), pages 203-228.

[7] J. Ginibre, Commun. Math. Phys. 8, 26 (1968). 
[8] E. H. Lieb, R. Seiringer, J. Yngvason, Phys. Rev. Lett. 94, 080401 (2005).

[9] A. Sütő, Phys. Rev. A. 71, 023602 (2005).

[10] A. Sütő, Phys. Rev. Lett. 94, 080402 (2005).

[11] V. A. Zagrebnov, J. B. Bru, Physics Reports 350, 291 (2001).

[12] J. I. Rivas, A. Camacho, E. Göklü, arXiv:1112.33.03.

[13] E. H. Lieb, R. Seiringer, J. P. Solvej, J. Yngvason, The Mathematics of the Bose Gas and its Condensation, Birkäuser, (2005).

[14] L. Pitaevskii, S. Stringari, Bose-Einstein Condensation, Oxford University Press (2003).

[15] C. J. Pethick, H. Smith, Bose-Einstein Condensation in Dilute Gases, Cambridge University Press (2002).

[16] J. S. Dowker, G. Kennedy, J. Phys. A: Math. Gen. 11, 895 (1978).

[17] J. S. Dowker, J. P. Schofield, Nucl. Phys. B327, 267 (1989).

[18] D. J. Toms, Phys. Rev. Lett. 69, 1152 (1992).

[19] D. J. Toms, Phys. Rev. D47, 2483 (1993).

[20] K. Kirsten, D. J. Toms, Phys. Rev. D55, 7797 (1997).

[21] F. Jüttner, Z. Phys. 47, 542 (1928).

[22] W. Glaser, Z. Phys. 94, 677 (1935).

[23] P. T. Landsberg, J. Dunning-Davis, Phys. Rev. 138, 1049 (1965).

[24] M. M. Nieto, Lett. Nuovo Cimento, 1, 677 (1969); J. Math. Phys. 11, 1346 (1970).

[25] M. B. Al’taie, J. Phys. A11, 1603 (1978).

[26] R. Beckmann, F. Karsch, D. E. Miller, Phys. Rev. Lett. 43, 1277 (1979).

[27] C. A. A. de Carvalho, S. G. Rosa Jr., J. Phys. A13, 989 (1980).

[28] R. Beckmann, F. Karsch, D. E. Miller, Phys. Rev. A25, 561 (1988).

[29] H. E. Haber, H. A. Weldon, Phys. Rev. Lett. 46, 1497 (1981). 
[30] H. E. Haber, H. A. Weldon, Phys. Rev. D25, 502 (1982).

[31] L. Burakovsky, L. P. Horwitz, W. C. Schrive, Phys. Rev. D54, 4029 (1996).

[32] A. Filippi, arXiv:hep-ph/9703323.

[33] R. Hakim, Introduction to Relativistic Statistical Mechanics, World Scientific (2011).

[34] P. Li, S. T. Yau, Acta Mathematica 156, 153, (1986).

[35] B. Colbois, D. Maerten, arXiv 0802.2774v1. [math.DG].

[36] P. C. Hohenberg, Phys. Rev. 158, 383 (1967).

[37] G. Roepstorff, J. Stat. Phys. 18, 191 (1978).

[38] O. Penrose, Phys. Lett. 11, 224 (1964).

[39] L. P. Pitaevskii, JETP Lett. 45, 185 (1987).

[40] L. P. Pitaevskii, S. Stringari, J. Low Temp. Phys. 85, 377 (1991).

[41] A. L. Leggett, New Journal of Physics 3, 23.1 (2001).

[42] G. Fucci, K. Kirsten, J. Phys. A44 332002 (2011).

[43] K. Kirsten, D. J. Toms, Phys. Rev. D51 6886 (1995).

[44] H. Umezawa, Advanced Quantum Field Theory, Micro, Macro and Thermal Physics, AIP (1993).

[45] I. S. Gradshteyn, I. M. Ryzhik, A. Jeffrey, D. Zwillinger, Table of Integrals, Series, and Products, Academic Press (2000).

[46] K. Kirsten and D. J. Toms, Phys Rev E59 158 (1999).

[47] T. P. Branson, P. B. Gilkey, Comm. Partial Differential Equations 15 245 (1990).

[48] J. Wang, Pacific Journal of Mathematics 178, 377, (1997).

[49] F. A. Berezin, Math. USSR Izv. 6, 117 (1972).

[50] F. A. Berezin, Commun. Math. Phys. 40, 153 (1975).

[51] B. Simon, Commun. Math. Phys. 71, 247 (1980). 
[52] B. Baumgartner, Commun. Math. Phys. 75, 25 (1980).

[53] E. H. Lieb, Commun. Math. Phys. 31, 327 (1973).

[54] J. Wang, Pacific Journal of Mathematics, 178, 377 (1997).

[55] I. Chavel, Eigenvalues in Riemannian Geometry, Academic Press (1984).

[56] H. Donnelly, P. Li, Michigan Math. J. 29, 149 (1982).

[57] A. P. Prudnikov, Y. A. Brychkov, O. I. Marichev, Integrals and Series Vol. 2: Special Functions, Gordon and Breach (1986).

[58] N. D. Mermin, H. Wagner, Phys. Rev. Lett. 17, 1133 (1966).

[59] M. Abramowitz, I. A. Stegun, Handbook of Mathematical Functions, Dover (1972). 